Hans-Görg Roos

Martin Stynes

Lutz Tobiska

\title{
Robust Numerical Methods for Singularly Perturbed Differential Equations
}

Convection-Diffusion-Reaction and Flow Problems

Second Edition

With 41 Figures

et Springer 


\section{Contents}

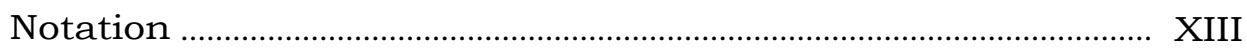

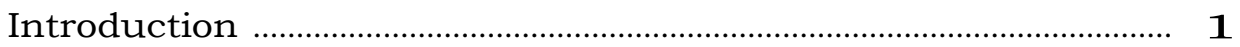

Part I Ordinary Differential Equations

1 The Analytical Behaviour of Solutions ......................................... 9

1.1 Linear Second-Order Problems Without Turning Points ........... 11

1.1.1 Asymptotic Expansions ..................................................... 12

1.1.2 The Green's Function and Stability Estimates ................ 16

1.1.3 A Priori Estimates for Derivatives and Solution

Decomposition ……………………................................... 21

1.2 Linear Second-Order Turning-Point Problems .............................. 25

1.3 Quasilinear Problems ...................................................................... 29

1.4 Linear Higher-Order Problems and Systems ………………….... 35

1.4.1 Asymptotic Expansions for Higher-Order Problems 35

1.4.2 A Stability Result ................................................................. 36

1.4.3 Systems of Ordinary Differential Equations .................... 38

2 Numerical Methods for Second-Order Boundary Value

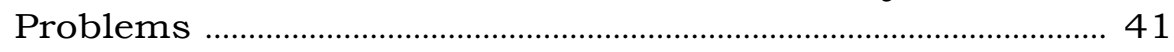

2.1 Finite Difference Methods on Equidistant Meshes ....................... 41

2.1.1 Classical Convergence Theory for Central

Differencing .............................................................................. 41

2.1.2 Upwind Schemes ................................................................. 45

2.1.3 The Concept of Uniform Convergence ............................... 57

2.1.4 Uniformly Convergent Schemes of Higher Order ............. 66

2.1.5 Linear Turning-Point Problems ………………………….... 68

2.1.6 Some Nonlinear Problems .................................................. 71

2.2 Finite Element Methods on Standard Meshes .............................. 76

2.2.1 Basic Results for Standard Finite Element Methods . $\quad 76$ 
2.2.2 Upwind Finite Elements …………………………….......... 79

2.2.3 Stabilized Higher-Order Methods ………………………... 84

2.2.4 Variational Multiscale and Differentiated Residual Methods .............................................................................. 95

2.2.5 Uniformly Convergent Finite Element Methods .............. 104

2.3 Finite Volume Methods ...................................................................... 114

2.4 Finite Difference Methods on Layer-adapted Grids ..................... 116

2.4.1 Graded Meshes ..................................................................... 119

2.4.2 Piecewise Equidistant Meshes ........................................... 127

2.5 Adaptive Strategies Based on Finite Differences .......................... 141

Part II Parabolic Initial-Boundary Value Problems in One Space Dimension

$1 \quad$ Introduction ................................................................................ 155

2 Analytical Behaviour of Solutions ..................................................... 159

2.1 Existence, Uniqueness, Comparison Principle ............................ 159

2.2 Asymptotic Expansions and Bounds on Derivatives ................... 161

$3 \quad$ Finite Difference Methods ................................................................. 169

3.1 First-Order Problems ..................................................................... 169

3.1.1 Consistency ........................................................................ 169

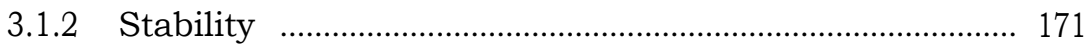

3.1.3 Convergence in L2 ............................................................ 174

3.2 Convection-Diffusion Problems .......................................................... 177

3.2.1 Consistency and Stability …………………..................... 178

3.2.2 Convergence ....................................................................... 182

3.3 Polynomial Schemes …………………………………………...... 183

3.4 Uniformly Convergent Methods ………………………………..... 187

3.4.1 Exponential Fitting in Space .............................................. 188

3.4.2 Layer-Adapted Tensor-Product Meshes .............................. 189

3.4.3 Reaction-Diffusion Problems ................................................. 191

$4 \quad$ Finite Element Methods ...................................................................... 195

$4.1 \quad$ Space-Based Methods …………………………………………….... 196

4.1.1 Polynomial Upwinding ....................................................... 197

4.1.2 Uniformly Convergent Schemes ............................................ 199

4.1.3 Local Error Estimates .......................................................... 203

4.2 Subcharacteristic-Based Methods ……………………………...... 205

4.2.1 SDFEM in Space-Time ..................................................... 206

4.2.2 Explicit Galerkin Methods .................................................. 211

4.2.3 Eulerian-Lagrangian Methods .............................................. 217 
5 Two Adaptive Methods 223

5.1 Streamline Diffusion Methods …………………………………...... 223

5.2 Moving Mesh Methods (r-refinement) .............................................. 225

\section{Part III Elliptic and Parabolic Problems in Several Space Dimensions}

1 Analytical Behaviour of Solutions ................................................... 235

1.1 Classical and Weak Solutions ………………………………..... 235

1.2 The Reduced Problem ...................................................................... 238

1.3 Asymptotic Expansions and Boundary Layers ………………...... 243

1.4 A Priori Estimates and Solution Decomposition .......................... 247

2 Finite Difference Methods ................................................................. 259

2.1 Finite Difference Methods on Standard Meshes ………………... 259

2.1.1 Exponential Boundary Layers ............................................. 259

2.1.2 Parabolic Boundary Layers ………………………………... 266

2.2 Layer-Adapted Meshes ………………………………………....... 268

2.2.1 Exponential Boundary Layers …………………………..... 268

2.2.2 Parabolic Layers ................................................................. 274

3 Finite Element Methods .................................................................... 277

3.1 Inverse-Monotonicity-Preserving Methods Based on Finite

Volume Ideas ……...................................................................... 278

3.2 Residual-Based Stabilizations …………………………………….. 302

3.2.1 Streamline Diffusion Finite Element Method

(SDFEM) …………........................................................... 302

3.2.2 Galerkin Least Squares Finite Element Method

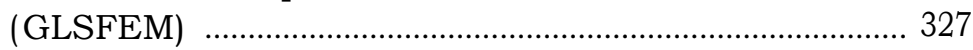

3.2.3 Residual-Free Bubbles ..................................................... 333

3.3 Adding Symmetric Stabilizing Terms …………………………...... 338

3.3.1 Local Projection Stabilization .............................................. 338

3.3.2 Continuous Interior Penalty Stabilization ……………..... 352

3.4 The Discontinuous Galerkin Finite Element Method .................. 363

3.4.1 The Primal Formulation for a Reaction-Diffusion

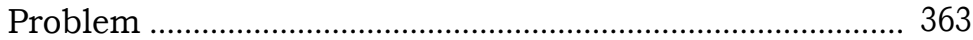

3.4.2 A First-Order Hyperbolic Problem ................................... 368

3.4.3 dGFEM Error Analysis for Convection-Diffusion
Problems

3.5 Uniformly Convergent Methods ………………………………….... 376

3.5.1 Operator-Fitted Methods …………………………………... 377

3.5.2 Layer-Adapted Meshes ......................................................... 381

3.6 Adaptive Methods ............................................................................ 407

3.6.1 Adaptive Finite Element Methods for Non-Singularly Perturbed Elliptic Problems: an Introduction ................. 407 
3.6.2 Robust and Semi-Robust Residual Type Error Estimators

3.6.3 A Variant of the DWR Method for Streamline

Diffusion

4 Time-Dependent Problems

4.1 Analytical Behaviour of Solutions ................................................. 428

4.2 Finite Difference Methods ................................................................ 429

4.3 Finite Element Methods …................................................................ 434

Part IV The Incompressible Navier-Stokes Equations

1 Existence and Uniqueness Results .............................................. 449

2 Upwind Finite Element Method ............................................................... 453

3 Higher-Order Methods of Streamline Diffusion Type ........... 465

3.1 The Oseen Problem .............................................................................. 466

3.2 The Navier-Stokes Problem ............................................................ 476

4 Local Projection Stabilization for Equal-Order

Interpolation ...................................................................................................... 485

4.1 Local Projection Stabilization in an Abstract Setting ................ 486

4.2 Convergence Analysis ..................................................................... 488

4.2.1 The Special Interpolant ...................................................... 488

4.2.2 Stability ........................................................................... 489

4.2.3 Consistency Error ............................................................... 491

4.2.4 A priori Error Estimate ....................................................... 492

4.3 Local Projection onto Coarse-Mesh Spaces ................................... 498

4.3.1 Simplices .......................................................................... 498

4.3.2 Quadrilaterals and Hexahedra ............................................. 499

4.4 Schemes Based on Enrichment of Approximation Spaces .......... 501

4.4.1 Simplices ............................................................................... 502

4.4.2 Quadrilaterals and Hexahedra ............................................ 502

4.5 Relationship to Subgrid Modelling ................................................... 504

4.5.1 Two-Level Approach with Piecewise Linear Elements .. 505

4.5.2 Enriched Piecewise Linear Elements ............................... 507

4.5.3 Spectral Equivalence of the Stabilizing Terms on Simplices ....................................................................... 508

5 Local Projection Method for Inf-Sup Stable Elements ......... 511

5.1 Discretization by Inf-Sup Stable Elements ................................ 512

5.2 Stability and Consistency ................................................................ 514

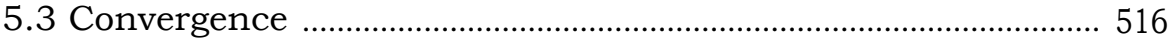

5.3.1 Methods of Order $\mathrm{r}$ in the Case $\mathrm{cr}>0$............................ 517

5.3.2 Methods of Order $r$ in the Case $o>0$............................... 522

5.3.3 Methods of Order $\mathrm{r}+1 / 2$............................................. 526 


\section{Mass Conservation for Coupled Flow-Transport}

Problems

6.1 A Model Problem ................................................................................. 529

6.2 Continuous and Discrete Mass Conservation .............................. 530

6.3 Approximated Incompressible Flows .......................................... 532

6.4 Mass-Conservative Methods ............................................................. 534

6.4.1 Higher-Order Flow Approximation ................................. 534

6.4.2 Post-Processing of the Discrete Velocity .......................... 536

6.4.3 Scott-Vogelius Elements .................................................. 542

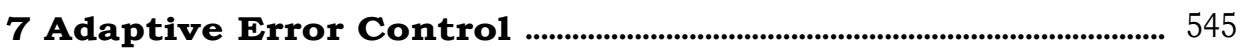

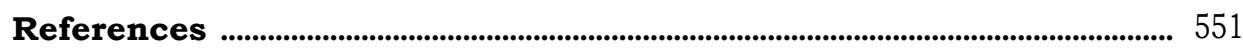

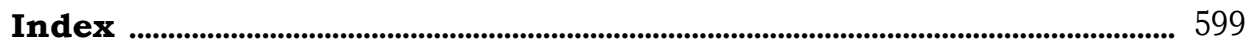

